\title{
Solid-State interferometric interrogator and multiplexer for high-speed dynamic and absolute FBG wavelength measurement
}

\author{
Philip Orr, Marcus Perry, Grzegorz Fusiek and Paweł Niewczas \\ Institute for Energy and Environment, Department of Electronic and Electrical Engineering, \\ University of Strathclyde, Glasgow G1 1XW, United Kingdom \\ Tel: +44 (0)141548 4841, Fax: +44 (0)141548 4872 \\ E-mail: philip.orr@strath.ac.uk
}

\begin{abstract}
We present a solid-state FBG array interrogator and multiplexer capable of determining absolute FBG wavelengths and of providing high-speed, high-resolution static and dynamic measurements. Using a described procedure, deployable on multiplexing passive-interferometric schemes, the system is able to determine initial sensor wavelengths and thereafter track wavelength changes with interferometric resolution. The scheme allows high-resolution interrogation of FBG sensor arrays to be applied to many industrial applications, where previously the lack of combined absolute and quasistatic wavelength measurement precluded the use of interferometric techniques. Using a preliminary laboratory embodiment, we demonstrate a wavelength determination accuracy of $<0.3 \mathrm{~nm}$ and a measurement resolution of 10 $\mathrm{fm} / \sqrt{ } \mathrm{Hz}$, and propose pathways to improved performance and miniaturisation.
\end{abstract}

Key words: fibre Bragg gratings, absolute wavelength, interrogation systems, sensor multiplexing

\section{INTRODUCTION}

Fibre Bragg grating (FBG) sensors are now ubiquitous in the field of optical instrumentation, primarily due to their robustness, low cost, high performance, and simplicity of interrogation and multiplexing ${ }^{1,2}$. However, the performance of an FBG sensor array, such as the achievable interrogation rate, accuracy, and resolution of each discrete sensor, is determined primarily by the interrogation system deployed. While FBG wavelength measurement has been realised by a number of methods (including interferometry and prism/CCD arrays ${ }^{3}$ ), arguably the most common uses a combination of a broadband light source, a tunable filter (either scanning Fabry-Perót or acousto-optic ${ }^{4}$ ) and a photodetector. In such 'tuned' schemes, multiplexing is achieved relatively straightforwardly at the expense of measurement resolution and rate. This trade-off is generally justified, however, by the ability of these sensors to provide an absolute measure of FBG wavelengths, as this is a pre-requisite for the deployment of FBG sensor arrays in many industrial applications.

While interferometric interrogation of FBGs offers the highest resolution of all techniques, it has traditionally been difficult to integrate with existing multiplexing schemes due to its requirement for active locking or scanning of the operating point. Recent passive-interferometric FBG interrogators are beginning to address this issue ${ }^{5,6}$, but the absolute accuracy of these schemes remains limited due to the cyclic nature of phase demodulation. In this paper, we detail the preliminary development and testing of an interferometric FBG interrogation scheme capable of providing a measure of absolute FBG wavelengths, facilitating static and absolute measurements for applications such as pressure or temperature monitoring $^{7}$. Crucially, this system retains the capability for high-speed dynamic measurements over the sensor array without a loss of interferometric resolution or large-scale sensor multiplexing capability. The scheme will therefore aid FBG sensing in becoming applicable to a broad range of industrial measurement applications. We firstly detail the system design before proceeding to demonstrate the performance both through simulation and using a preliminary laboratory embodiment. Finally, we discuss ways by which the performance may be improved through further development, and make recommendations for system miniaturisation, fabrication, and packaging. 


\section{SYSTEM DESIGN}

The proposed sensor interrogation scheme is illustrated in Fig. 1(a). A broadband source illuminates the sensor array, within which each FBG reflects at a unique wavelength $\lambda_{1}-\lambda_{\mathrm{n}}$. Sensor FBG reflections are coupled into a series of demodulation elements to enable multiplexing, wavelength determination, and wavelength tracking. Wavelength division multiplexing is achieved at high speed using a technique described previously ${ }^{6}$, whereby an optical wavelength switch comprising an arrayed-waveguide grating (AWG) and optical path switch are utilised to switch (with a transition time of the order of ns) between flat-top spectral bins that are assigned uniquely to each sensor in the array. Individual sensor reflections are therefore time-sequenced and delivered serially to an asymmetrical Mach-Zehnder interferometer (AMZI) that is thermally stabilised and incorporates the capability for brief tuning of a single path.

By utilising an AMZI terminating in a $3 \times 3$ coupler at the point of interference, a passive, ratiometric phase-demodulation algorithm such as Todd's ${ }^{8}$ may be deployed in order to continually track phase differences while retaining immunity to fluctuations in input optical power or attenuation en route to sensors. In the preferred embodiment, the immediacy of the switching multiplexer may be exploited in order to perform oversampling of each FBG reflection, enabling anti-alias filtering for noise reduction after ADC by synchronously driving the sampling clock at an integer multiple of the bandswitching clock. The proposed architecture therefore allows for multiplexing rates limited by switching speed and an oversampling factor limited by ADC capabilities.

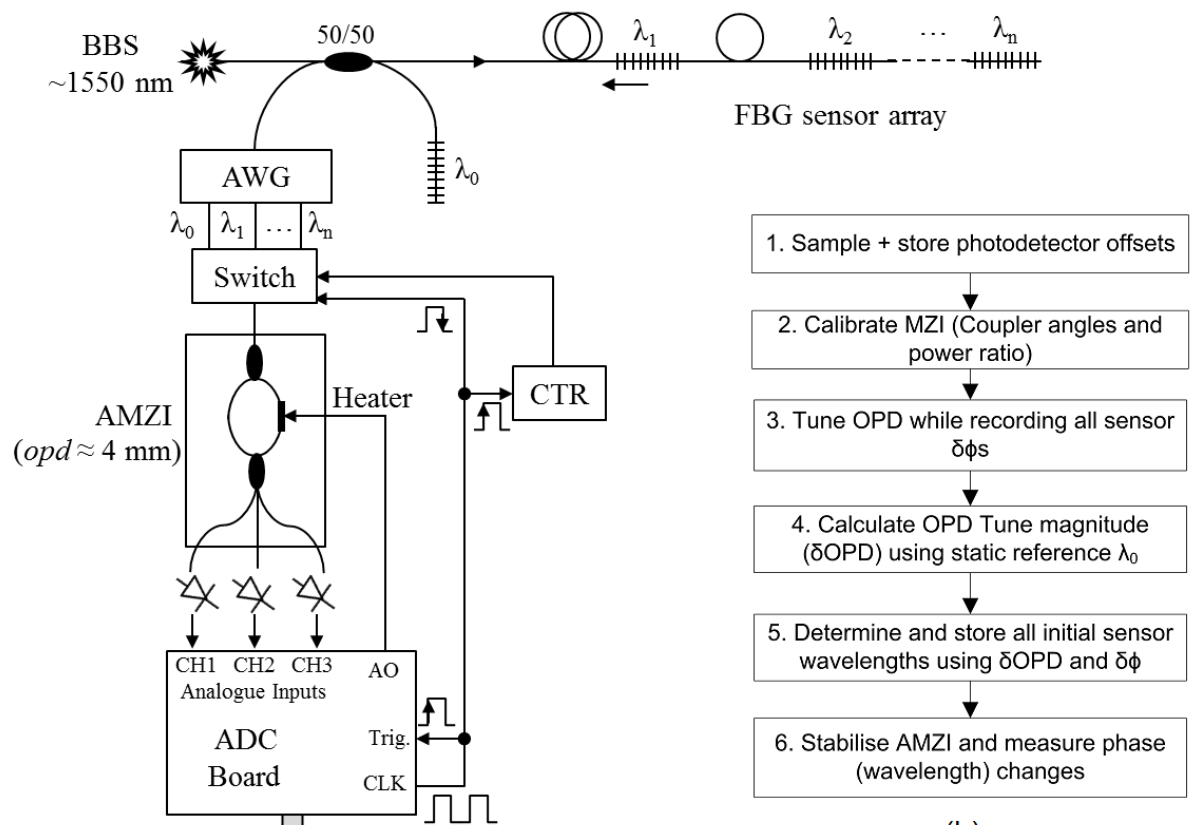

(a)

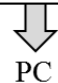

(b)

Figure 1. (a) Proposed FBG interrogator and multiplexer. AWG is arrayed waveguide grating, AMZI is asymmetric Mach-Zehnder interferometer, BBS is broadband source. (b) Procedure for interrogator initialisation and determination of initial sensor wavelengths.

Key to providing the capability for absolute FBG wavelength measurement is the initialisation procedure described in Fig. 1(b). The procedure relies on brief tuning of the AMZI path difference from its nominal unbalance (which thereafter is returned to a relaxed steady state for sensor wavelength tracking). During path unbalance tuning, implemented by a heater in the present embodiment, the multiplexing technique is used to monitor phase change on all FBG channels simultaneously - sensor FBGs and a single stable reference wavelength that is known to a sufficient degree of accuracy. For each sensor, there exists a unique ratio between the incurred phase change of the reference wavelength and that of the sensor wavelength. This is since the phase difference $\phi$ at the interferometer output is related to the input optical wavelength $\lambda$ by the expression 


$$
\phi=\frac{2 \pi n d}{\lambda}
$$

where $n$ is the MZI core index (tunable by heating) and $d$ is the physical path difference. For a reference at $\lambda_{\text {ref }}$ and a sensor at $\lambda_{1}$, the unique ratio relating the phase changes $\delta \phi_{\text {ref }}$ and $\delta \phi_{1}$ during an arbitrary path unbalance tuning of $\delta(n d)$ can be shown to be

$$
\frac{\delta \phi_{r e f}}{\delta \phi_{1}}=\frac{\lambda_{1}}{\lambda_{\text {ref }}}
$$

Which may be used straightforwardly to calculate $\lambda_{1}$ and all other sensor wavelengths in the same manner. This assumes that the sweep time is sufficiently less than any present perturbations on all sensors such that sensor wavelengths can be assumed to be stable during the procedure. It is clear from (2) that error is introduced both by uncertainty in the reference wavelength, and by noise in the measured values of the phase excursions.

\section{SIMULATION OF ABSOLUTE WAVELENGTH DETERMINATION}

To demonstrate the determination of absolute wavelength, a simulation has been constructed based on the system in Fig. 1(a) incorporating phase demodulation of sensor and reference wavelengths using Todd's passive algorithm ${ }^{8}$. Separate phase unwrapping and a phase noise floor of $100 \mu \mathrm{rad} / \sqrt{\mathrm{Hz}}$ on each sensor (corresponding to the noise floor of our laboratory scheme) are included. As with practical interferometric interrogators, the measurements are referenced (zeroed) to "switch on" time, and no absolute measurements are initially available. Reference and sensor wavelengths of $1560 \mathrm{~nm}$ and $1550 \mathrm{~nm}$, respectively, are simulated in this case.

To simulate the tuning of phase unbalance, an initial OPD of $4 \mathrm{~mm}$ is detuned by $1 \%$ at a rate of $50 \mu \mathrm{m} / \mathrm{s}-$ parameters which are straightforwardly achieved in planar interferometers 9 . As shown in Fig. 2(a), the OPD is held constant for 100 $\mathrm{ms}$ both prior to and following detuning, during which periods averaging is performed to yield the pre- and post-tuning phase differences (reducing the impact of phase noise). The calibration process therefore takes $0.5 \mathrm{~s}$ in total in this example - in practice, higher interrogation rates will worsen phase noise but will allow for an increase in tuning rate and hence reduce initialisation time. An interrogation rate of $1 \mathrm{kHz}$ is utilised in this simulation, which is sufficient to correctly process wrapping phases at a tuning rate of $50 \mu \mathrm{m} / \mathrm{s}$ without discontinuities arising.

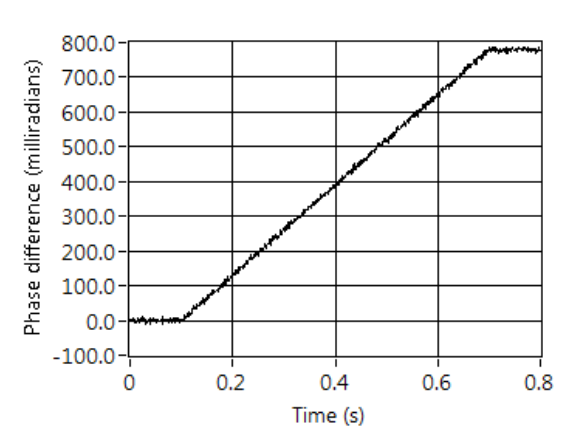

(a)

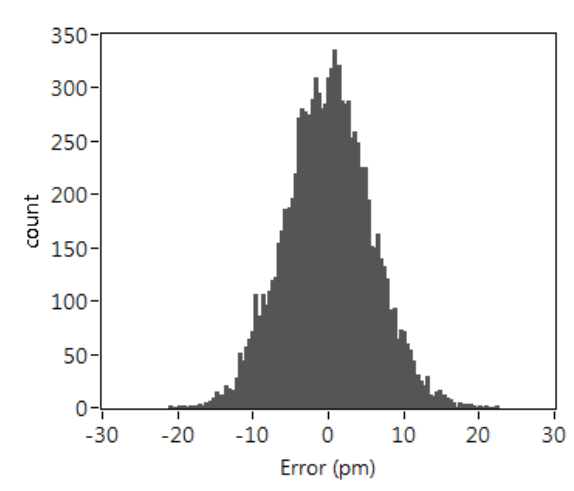

(b)

Figure 2. (a) Incurred difference between phase excursions in reference and sensor wavelength during OPD tuning. Tuning rate 50 $\mu \mathrm{m} / \mathrm{s}$, total detuning $40 \mu \mathrm{m}$ ( $1 \%$ of initial OPD), interrogation rate $1 \mathrm{kHz}$. (b) Histrogram (10,000 iterations) of sensor wavelength determination error. Standard deviation is $5.61 \mathrm{pm}$.

Fig. 2(b) illustrates the error in sensor wavelength calibration over 10,000 iterations of the procedure. Standard error in the wavelength is $5.61 \mathrm{pm}$, corresponding to absolute measurement accuracies of $0.43{ }^{\circ} \mathrm{C}$ or $4.87 \mu \varepsilon$. It is worth noting that inaccuracy of the reference wavelength translates directly to a comparable error in all sensor wavelengths. In practice however, temperature-stabilised athermal FBGs can achieve a stability of $<1 \mathrm{pm}^{10}$. The more significant 
influence of phase noise can be reduced by increasing the span between reference and sensor wavelengths, which will produce a larger differential phase $\left(\delta \phi_{\text {ref }}-\delta \phi_{\mathrm{n}}=2 \pi \cdot \delta \mathrm{OPD} \cdot\left(1 / \lambda_{\text {ref }}-1 / \lambda_{\mathrm{n}}\right)\right)$, and hence a larger signal-to-noise ratio.

\section{PRELIMINARY LABORATORY TESTS}

In order to demonstrate practically the performance of the proposed scheme (both in terms of absolute accuracy, and of static and dynamic resolution), the system in Fig. 1(a) has been constructed with a limited number of multiplexed sensors. For comparison with the simulation in Section 3, an athermal grating (without active temperature control) is utilised as the wavelength reference $\lambda_{\text {ref }}$ located at $1559.96 \mathrm{~nm}$. A sensing FBG located at $1549.14 \mathrm{~nm}$ is mounted on a cantilever, which was stabilised during the initialisation process. The AMZI, incorporating a path difference of $4.58 \mathrm{~mm}$, is an all-fibre construction fabricated between the output pigtails of $1 \times 2$ and $3 \times 3$ couplers by fusion splicing. To isolate the MZI from vibration and to hinder heat transfer during tuning, the device is bonded to a glass slab by epoxy. Thermal tuning of the OPD is facilitated by a resistive heater in thermal contact with a single MZI arm. The ADC unit is configured to sample at $4 \mathrm{kHz}$ with an oversampling factor of 4 , thus providing a measurement rate of $0.5 \mathrm{kHz}$ per sensor.
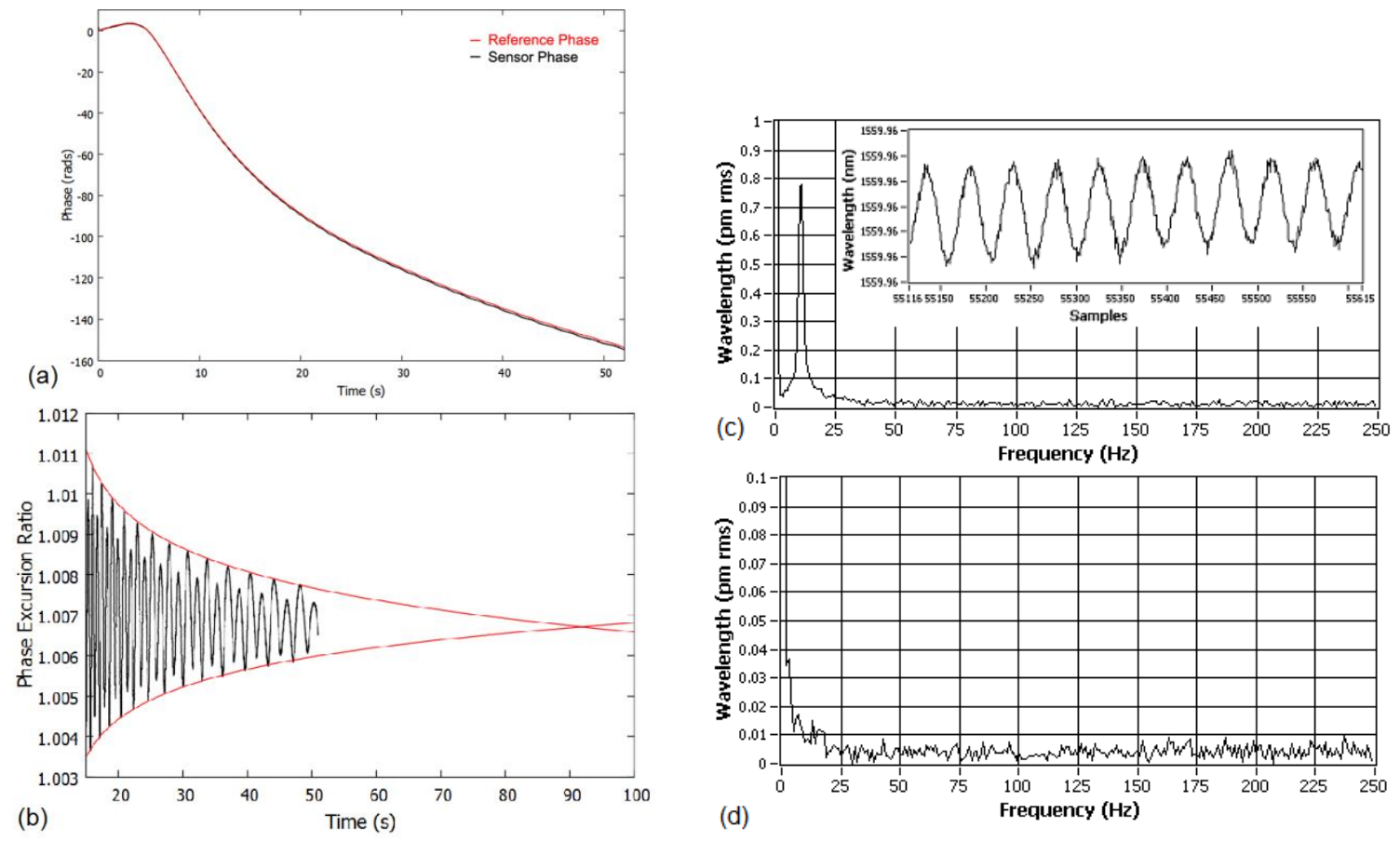

Figure 3. (a) Phase excursions of sensor and reference FBG reflections during OPD tuning ( $1 \%$ of initial OPD, i.e. $40 \mu \mathrm{m})$. (b) Convergence of sensor wavelength determination process on correct $\lambda_{1} / \lambda_{\text {ref }}$ ratio of 1.006598 . (c) Amplitude spectrum and time plot (inset) of sensor wavelength during small $(\sim 5 \mathrm{pm} \mathrm{rms}) 10 \mathrm{~Hz}$ perturbation of cantilever. (d) Spectral noise floor $(<10 \mathrm{fm} / \sqrt{ } \mathrm{Hz})$ of system recorded using athermal reference FBG.

An OPD detuning of approximately 140 rads (or 1\%) is produced during this experiment, allowing direct comparison with the simulation in Section 3. Fig. 3(a) shows the phase excursions of reference and sensor FBGs during OPD tuning, while Fig. 3(b) illustrates the convergence of the calculated ratio $\delta \phi_{\text {ref }} / \delta \phi_{1}$. It can be seen that the ratio calculation converges on the ideal value of 1.00659 for the particular reference and sensor wavelengths utilised in this experiment. A repeatable accuracy of $<0.3 \mathrm{~nm}$ has been achieved in this experiment by determining the unique ratio through averaging, which can be improved in future embodiments by using different techniques as discussed in the following section.

Following initialisation - and, in this preliminary embodiment, allowing sufficient time for the AMZI to re-stabilise measurement data are recorded to illustrate the dynamic and static performance of the interrogator. In this test, a small perturbation is applied to the sensing cantilever, which resonates naturally at $10 \mathrm{~Hz}$, producing a $\sim 5 \mathrm{fm} \mathrm{rms}$ wavelength 
shift (Fig. 3(c)). Simultaneously, a noise spectral density of $<10 \mathrm{fm} / \sqrt{ } \mathrm{Hz}$ is visible on the athermal reference grating (Fig. $3(d))$.

\section{DISCUSSION}

As discussed by the authors previously ${ }^{11}$, there is an inherent nonlinearity associated with the passive interferometric phase demodulation technique utilised here, which becomes noticeable only under conditions of very large signals. A nonlinear sensitivity is produced as FBG reflections pass over fringe maxima and minima and are "pulled" slightly towards the maxima locations. In this work, the large OPD tuning magnitude induced to allow calculation of initial sensor wavelengths gives rise to such oscillations in the converging ratio calculation of Fig. 3(b). As the phase excursions of the reference and sensor FBGs diverge, the impact of the nonlinear responses lessens, allowing the calculation of sensor wavelengths to be performed regardless. However, there is clear potential for further work in improving the speed and accuracy of the ratio estimation using signal processing techniques, by reducing the linewidth of the FBGs, or by utilising fibre laser sensors.

Due to the solid-state nature of the proposed system, its miniaturised implementation may be achieved using a silicon photonics platform whereby the AWG, switching, tunable AMZI, and photodetection elements of Fig. 1(a) may be incorporated on a single silicon chip ${ }^{12}$. Given the tremendous growth in this field at present, it is likely that low-cost mass production of such interrogators will be best facilitated in this manner.

\section{CONCLUSIONS}

In this paper we have described an FBG interrogator and multiplexer that combines absolute sensor wavelength determination with interferometric resolution for static and dynamic measurements. An initialisation procedure for utilising a switched-band wavelength multiplexing technique to determine the starting wavelengths of all multiplexed sensors was described, simulated, and demonstrated practically. A preliminary laboratory demonstration of the system has been shown to be capable of an absolute wavelength determination accuracy of $<0.3 \mathrm{~nm}$ and thereafter a wavelength tracking resolution of $<10 \mathrm{fm} / \sqrt{ } \mathrm{Hz}$ for static and dynamic measurements, and is therefore likely to be applicable to a wide range of demanding industrial measurement applications. The performance of the wavelength determination technique was discussed and shown to be improvable through future work focussed on signal processing, the reduction of sensor linewidths, and implementation of the scheme on a silicon photonics platform.

\section{ACKNOWLEDGEMENTS}

This work was supported by the Engineering and Physical Sciences Research Council.

\section{REFERENCES}

[1] Kersey, A. D. et al, "Fiber Grating Sensors," J. Lightwave Tech. 15(8), 1442-1463 (1997)

[2] Mendez, A., "Fiber Bragg grating sensors: a market overview," Proc. SPIE 6619, p. 661905 (2007)

[3] Ezbiri, A., Kanellopoulos, S. E., and Handerek, V. A., "High resolution instrumentation system for fibre-Bragg grating aerospace sensors," Opt. Comm. 150, 43-48 (1998)

[4] Boulet, C., Webb, D. J., Douay, M., and Niay, P., "Simultaneous interrogation of fiber Bragg grating sensors using an acoustooptic tunable filter," Phot. Tech. Lett. 13(10), 1215-1217 (2001)

[5] Johnson, G. A., Todd, M. D., Aulthouse, B. L. and Chang, C. C., "Fiber Bragg grating interrogation and multiplexing with a $3 \times 3$ coupler and a scanning filter," J. Lightwave Tech. 18(8), 1101-1105 (2000)

[6] Orr, P. and Niewczas, P., "High-speed, solid-state, interferometric interrogator and multiplexer for fiber Bragg grating sensors," J. Lightwave Tech. 29(22), 3387-3392 (2011)

[7] Majumder, M., Gangopadhyay, T. K., Chakraborty, A. K., Dasgupta, K., Bhattacharya, D. K., "Fibre Bragg gratings in structural health monitoring - Present status and applications," Sens. Act. A: Phys. 147, 150-164 (2008)

[8] Todd, M. D., Johnson, G. A. and Chang, C. C., "Passive light intensity-independent interferometric method for fibre Bragg grating interrogation," Elec. Lett. 35(22), 1970-1971 (1999)

[9] Holmes, C., Kundys, D. O., Gates, J. C., Gawith, C. B. E. and Smith, P. G. R., "150 GHz of thermo-optic tuning in direct UV written silica-on-silicon planar Bragg grating," Elec. Lett. 45(18), 954-955 (2009) 
[10] Lo, Y. L. and Kuo, C. P., "Packaging a fiber Bragg grating without preloading in a simple athermal bimaterial device," IEEE Trans. Advanced Packaging 25(1), 50-53 (2002)

[11] Orr, P. and Niewczas, P., "Passive, Multiplexable Measurement of Magnetic Field Strength and Temperature Using PM Fibre Bragg Gratings and Chiral Fibre Devices," Proc. $20^{\text {th }}$ Int. Conf. on Optical Fiber Sensors (2009)

[12] Jalali, B., Fathpour, S., "Silicon Photonics," J. Lightwave Tech. 24(12), 4600-4615 (2006) 